\title{
Observation of the fastest chemical processes in the radiolysis of water
}

Z.-H. Loh, ${ }^{1 *}$ G. Doumy, ${ }^{2}$ C. Arnold,,${ }^{3,4,5}$ L. Kjellsson, ${ }^{6,7}$ S.H. Southworth, ${ }^{2}$ A. Al Haddad, ${ }^{2}$ Y. Kumagai, ${ }^{2}$ M.-F. Tu, ${ }^{2}$ P.J. Ho, ${ }^{2}$, A.M. March,${ }^{2}$ R.D. Schaller, ${ }^{8,9}$ M.S. Bin Mohd Yusof, ${ }^{1}$ T. Debnath, ${ }^{1}$ M. Simon, ${ }^{10}$ R. Welsch ${ }^{3,5}$ L. Inhester, ${ }^{3}$ K. Khalili, ${ }^{11}$ K. Nanda, ${ }^{12}$ A.I. Krylov, ${ }^{12}$ S. Moeller, ${ }^{13}$ G. Coslovich, ${ }^{13}$ J. Koralek, ${ }^{13}$ M.P. Minitti, ${ }^{13}$ W.F. Schlotter, ${ }^{13}$ J.-E. Rubensson, ${ }^{6}$, R. Santra, ${ }^{3,4,5 *}$ L. Young ${ }^{2,14 *}$

${ }^{1}$ Division of Chemistry and Biological Chemistry, School of Physical and Mathematical Sciences, Nanyang Technological University, Singapore

${ }^{2}$ Chemical Sciences and Engineering Division, Argonne National Laboratory, Lemont, IL USA

${ }^{3}$ Center for Free-Electron Laser Science, DESY, Hamburg, Germany

${ }^{4}$ Department of Physics, Universität Hamburg, Hamburg, Germany

${ }^{5}$ The Hamburg Centre for Ultrafast Imaging, Hamburg, Germany

${ }^{6}$ Department of Physics and Astronomy, Uppsala University, Uppsala, Sweden

${ }^{7}$ European XFEL GmbH, Schenefeld, Germany

${ }^{8}$ Center for Nanoscale Materials, Argonne National Laboratory, Lemont, IL, USA

${ }^{9}$ Department of Chemistry, Northwestern University, Evanston, IL, USA

${ }^{10}$ Sorbonne Université and CNRS, Laboratoire de Chemie Physique-Matière et Rayonnement, LCPMR, F-750005, Paris, France

${ }^{11}$ Department of Energy Conversion and Storage,

Technical University of Denmark, Roskilde, Denmark

${ }^{12}$ Department of Chemistry, University of Southern California, Los Angeles, CA, USA

${ }^{13}$ LCLS, SLAC National Accelerator Laboratory, Menlo Park, CA, USA

${ }^{14}$ Department of Physics and James Franck Institute, The University of Chicago, Chicago, IL, USA

${ }^{*}$ To whom correspondence should be addressed;

E-mail: zhiheng@ntu.edu.sg, robin.santra@cfel.de, young@anl.gov 
One sentence summary: Ultrafast x-rays capture the elementary steps of proton transfer and hydroxyl radical solvation in ionized liquid water.

Elementary processes associated with ionization of liquid water provide a framework for understanding radiation-matter interactions in chemistry and biology. While numerous studies have been conducted on the dynamics of the hydrated electron, its partner arising from ionization of liquid water, $\mathrm{H}_{2} \mathrm{O}^{+}$, remains elusive. We use tunable femtosecond soft $x$-ray pulses from an x-ray free electron laser to reveal the dynamics of the valence hole created by strongfield ionization and to track the primary proton transfer reaction giving rise to the formation of $\mathrm{OH}$. The isolated resonance associated with the valence hole $\left(\mathrm{H}_{2} \mathrm{O}^{+} / \mathrm{OH}\right)$ enables straightforward detection. QM/MM calculations reveal that the $x$-ray spectra are sensitive to structural dynamics at the ionization site. We find signatures of hydrated-electron dynamics in the x-ray spectrum.

Radiolysis of liquid water is a universal phenomenon that accompanies the interaction of high-energy radiation with matter in aqueous environments. It is of fundamental importance in many domains ( 1 ), ranging from water-cooled nuclear reactors where radiolysis products cause corrosion (2) to radiation-induced genomic damage in living organisms where water comprises $80 \%$ by weight (3), thereby making radiolysis foundational to medical treatment, diagnosis and even extended human space flight (4). While ionizing radiation is delivered via various vehicles (x-rays, $\gamma$-rays, charged particles) its interaction with matter can be understood conceptually as individual absorption events along the particle path accompanied by a cascade of electrons, ions and radicals (5).

Consider the most elementary process: ionization of pure liquid water which leads to the 
formation of a hydrated electron precursor and a cationic hole $\left(\mathrm{H}_{2} \mathrm{O}^{+}\right)$. Both species are very reactive. The dynamics of the hydrated electron, $e_{a q}^{-}$, has been the subject of numerous studies (6-11) since the discovery of its visible spectrum (12), which peaks at $718 \mathrm{~nm}$ and spans 500$1000 \mathrm{~nm}$ - convenient for ultrafast laser spectroscopies. In stark contrast, its ionization partner, $\mathrm{H}_{2} \mathrm{O}^{+}$, has not been experimentally detected. The $\mathrm{H}_{2} \mathrm{O}^{+}$is predicted to undergo rapid sub100 -fs proton transfer to a neighboring water molecule to yield the hydronium cation $\left(\mathrm{H}_{3} \mathrm{O}^{+}\right)$ and the hydroxyl radical $(\mathrm{OH})(1,14,15)$. Attempts to observe directly the $\mathrm{H}_{2} \mathrm{O}^{+}$cation using ultrafast visible or ultraviolet probes have been inconclusive due to its ultrashort lifetime and masked spectral signature $(6,13,15)$. Thus, basic questions regarding the ionization of water remain. What is the lifetime of $\mathrm{H}_{2} \mathrm{O}^{+}$? What are the absorption spectra of $\mathrm{H}_{2} \mathrm{O}^{+}$and $\mathrm{OH}$ ? What is the extent of hole delocalization in $\mathrm{H}_{2} \mathrm{O}^{+}$and its timescale for localization relative to proton transfer?

Here we introduce an ultrafast x-ray probe which enables us to track the primary chemical reaction following ionization of liquid water, namely $\mathrm{H}_{2} \mathrm{O}^{+}+\mathrm{H}_{2} \mathrm{O} \rightarrow \mathrm{OH}+\mathrm{H}_{3} \mathrm{O}^{+}$. Combined experiment and theory yield insight for the effort in this new spectral regime. X-rays are wellsuited for probing the short-lived $\mathrm{H}_{2} \mathrm{O}^{+}$cation and $\mathrm{OH}$ radical as their absorption lines fall cleanly in the water window. Removal of an electron from the outermost valence orbital $\left(1 b_{1}\right)$ of $\mathrm{H}_{2} \mathrm{O}$ produces a new transition for $\mathrm{H}_{2} \mathrm{O}^{+}$that is red-shifted from the $1 \mathrm{a}_{1} \rightarrow 4 \mathrm{a}_{1}$ pre-edge transition at $535 \mathrm{eV}$ (16) roughly by the HOMO-LUMO gap $\Delta E$ (17), as shown in Fig. 1A. The $\mathrm{OH}$ radical, isoelectronic to $\mathrm{H}_{2} \mathrm{O}^{+}$, possesses a nearby x-ray absorption resonance, while the other product of proton transfer, $\mathrm{H}_{3} \mathrm{O}^{+}$, has resonances that fall in a region of strong water absorption (Fig. 1B). The experimental configuration, consisting of a 60-fs, 800-nm strong-field ionization pump (10), tunable $\sim 30$ fs ultrafast x-ray probe from the LCLS (Linac Coherent Light Source) x-ray free-electron laser (XFEL) (18) and three photon-in/photon-out detection channels, is shown in Fig. 1C. 
Signatures of the impulsively produced valence hole and excess electron appeared in all three detection channels when the incident x-ray energy was scanned. Fig. 2A displays data from the dispersed fluorescence channel, whereas the transmission mode is used in Figs. 2B, $\mathrm{C}$ and D. Fig. 2A shows absorption prior to $(\Delta t<0 \mathrm{ps})$ and after ionization ( $\Delta t>100 \mathrm{fs})$. At negative time delay, the absorption is that of liquid water, i.e. nonresonant ionization of the valence and inner-valence levels of water plus the pre-edge transition. (Saturation effects prevented a clear observation of the pre-edge resonance.) At positive time delay, two new features are apparent: an absorption resonance at $525.9 \mathrm{eV}$ and a shift of the pre-edge absorption to lower energies. The new absorption resonance is consistent with the creation of a hole in the outermost valence level of liquid water. The corresponding $\mathrm{H}_{2} \mathrm{O}^{+}$transition energy can be estimated to be $526.9 \mathrm{eV}$ using the binding energies in liquid water of the $\mathrm{O} 1 \mathrm{~s}$ core level $(538.1 \mathrm{eV})(19)$ and the $1 \mathrm{~b}_{1} \mathrm{HOMO}(11.2 \mathrm{eV})(20)$. The isoelectronic $\mathrm{OH}$ exhibits a gas-phase absorption peak at $525.85 \mathrm{eV}(21)$.

The $\mathrm{H}_{2} \mathrm{O}^{+}$cation produced by the ionization of liquid water is widely expected to decay via a pseudo-first-order reaction involving proton transfer to a neighboring water molecule

$$
\mathrm{H}_{2} \mathrm{O}^{+}+\mathrm{H}_{2} \mathrm{O} \rightarrow \mathrm{OH}+\mathrm{H}_{3} \mathrm{O}^{+}
$$

to yield the $\mathrm{OH}$ radical and the $\mathrm{H}_{3} \mathrm{O}^{+}$species. In the absence of electron scavengers, the $\mathrm{OH}$ radical subsequently undergoes geminate recombination (22) with the ejected electron to give $\mathrm{OH}^{-}$. The time-resolved differential absorption spectrum, $\Delta A=A(\Delta t)-A(\Delta t<0)$, showed a prompt increase at time-zero, followed by a narrowing of the spectral width within the first picosecond, and finally a gradual decay at longer time delays (Fig. 2B). We modeled this behavior as sequential kinetics: the species initially produced by ionization decays with lifetime $\tau_{1}$ to give an intermediate species with lifetime $\tau_{2}$, with absorption spectra $S_{1}(E)$ and $S_{2}(E)$, respectively. 
We assign $S_{2}(E)$, obtained by averaging $\Delta A$ for time delays between $1.5-5.8 \mathrm{ps}$, to the $\mathrm{OH}$ radical (Fig. 2C). $S_{2}(E)$ can be fit to a sum of two Lorentzians, a main peak at $525.97 \pm 0.08$ $\mathrm{eV}$ and $\mathrm{a} \sim 7 \times$ weaker sideband at $526.45 \pm 0.12 \mathrm{eV}$. The $0.48-\mathrm{eV}$ energy spacing between the two peaks is in reasonable agreement with the $0.53-\mathrm{eV}$ spacing of the vibrational progression for core-excited gas-phase $\mathrm{OH}$ (21). Both peaks have a common FWHM (full width at half maximum) of $0.48 \pm 0.02 \mathrm{eV}$, significantly broader than the $0.1-\mathrm{eV}$ spectral bandwidth of the XFEL pulses and the $0.147-\mathrm{eV}$ natural linewidth (21), suggesting inhomogeneous broadening by the solvent environment.

With the x-ray absorption lineshape of the aqueous $\mathrm{OH}$ radical $S_{2}(E)$ determined, the next step is to extract the time constants $\tau_{1}$ and $\tau_{2}$, and the spectral lineshape of the short-lived component $S_{1}(E)$. We used a Gaussian instrument response function of 106-fs FWHM and performed a surface fit of the experimental data shown in Fig. 2B. (We did not convolve the kinetic model with the experimental energy resolution of $0.1-\mathrm{eV}$ FWHM because the experimentally observed spectral features were much broader.) The surface fit yielded $\tau_{1}=0.18 \pm 0.02 \mathrm{ps}$ and $\tau_{2}=14.2 \pm 0.4 \mathrm{ps}$ and a Lorentzian absorption lineshape $S_{1}(E)$ centered at $526.01 \pm 0.13$ $\mathrm{eV}$ with a FWHM of $0.98 \pm 0.04 \mathrm{eV}$. The decay of the $\mathrm{OH}$ radical, $\tau_{2}$, most likely originates from geminate recombination of the $\mathrm{OH}$ radical with a hydrated electron. The recombination time constant of $14.2 \pm 0.4 \mathrm{ps}$ is significantly shorter than those reported in the literature (22), most likely due to the high initial ionization fraction accelerating the geminate recombination process. Geminate recombination between $e_{a q}^{-}$and $\mathrm{OH}$ in ionized liquid water has been extensively investigated by time-resolved optical spectroscopy (22) and is beyond the scope of the present study. Nevertheless, considering the relative diffusion coefficients of the reactants and the reaction radius, our observed timescale for geminate recombination suggests an approximate ionization fraction of $1.7 \%$, in relatively good agreement with the ionization fraction estimated based on the $\mathrm{OH}$ x-ray absorbance (see below). The spectral lineshape $S_{1}(E)$ and 
time constant $\tau_{1}$ can be assigned either to the decay of $\mathrm{H}_{2} \mathrm{O}^{+}$or to the cooling of vibrationally hot $\mathrm{OH}$ radical. Both species should exhibit a larger spectral linewidth than the $\mathrm{OH}$ radical. The x-ray absorption linewidth of $\mathrm{H}_{2} \mathrm{O}^{+}$may be similar to the $1.5-\mathrm{eV}$-wide $1 b_{1}$ band in the valence photoelectron (20) and the x-ray emission spectra (23) of liquid water, whose widths have been attributed to a multitude of hydrogen-bonding configurations and intermolecular geometries. The x-ray absorption spectrum of vibrationally excited $\mathrm{OH}$ may be broadened due to the presence of additional hot-band transitions.

Further insight into the early-time dynamics was obtained with time traces at three selected photon energies: 525.43, 525.93, and $526.73 \mathrm{eV}$ (Fig. 2D). These time traces correspond to the isotropic signal $\Delta A_{i s o}=\left(\Delta A_{\|}+2 \Delta A_{\perp}\right) / 3$, reconstructed from measurements in which the relative polarizations of the pump and probe pulses are parallel $\left(\Delta A_{\|}\right)$and perpendicular $\left(\Delta A_{\perp}\right) . \Delta A_{\text {iso }}$ is independent of rotational reorientation of the transient species in solution and is therefore sensitive only to population dynamics. Importantly, and visible upon inspection, the $\Delta A_{\text {iso }}$ time trace for $525.93 \mathrm{eV}$ shows a significantly delayed rise relative to those for 525.43 and $526.73 \mathrm{eV}$. Attempts to perform a global fit with two time constants could not reproduce the early time dynamics (see SM, Supplementary Materials, for details). The presence of this delayed rise suggests the existence of an additional ultrafast process (24) that was not captured in our analysis of the $2 \mathrm{D}$ dataset shown in Fig. 2B. Indeed, global fitting of the $\Delta A_{\text {iso }}$ time traces revealed a new component with the time constant $46 \pm 10 \mathrm{fs}$, whereas the other two time constants extracted from the fit, $0.16 \pm 0.03 \mathrm{ps,} \mathrm{and} 9.2 \pm 1.3 \mathrm{ps}$, are comparable to those obtained from the analysis of the $2 \mathrm{D}$ dataset. This additional time constant appeared as a growth component at $525.93 \mathrm{eV}$ and to a smaller extent, at $526.73 \mathrm{eV}$ as well, hence accounting for their delayed appearances relative to the signal at $525.43 \mathrm{eV}$, which rose with the instrument response function where prompt absorption by vertically ionized water might be expected based upon the calculated relative positions of the $\mathrm{OH}$ and $\mathrm{H}_{2} \mathrm{O}^{+}$resonances. That the 
46-fs component manifested itself as a growing signal at $525.93 \mathrm{eV}$, near the peak position of the $\mathrm{OH}$ radical absorption maximum at $525.97 \mathrm{eV}$, strongly suggests that it reflects the timescale for the formation of the $\mathrm{OH}$ radical. Given that $\mathrm{OH}$ is formed from the decay of the $\mathrm{H}_{2} \mathrm{O}^{+}$radical cation, this interpretation would therefore suggest a lifetime of $46 \mathrm{fs}$ for the $\mathrm{H}_{2} \mathrm{O}^{+}$radical cation. The experiments were conducted at near-ambient temperature making them practically relevant. In this non-equilibrium situation, any local temperature rise does not affect the ultrafast proton transfer timescale.

To understand the observed ultrafast dynamics, we performed QM/MM excited-state molecular dynamics simulations of liquid water following strong-field ionization. We considered initial ionization in the upper $1.5 \mathrm{eV}$ of the valence band and averaged across 107 initial geometries of liquid water. Non-Born-Oppenheimer effects were taken into account by Tully's fewestswitches surface hopping approach (25). We combined a quantum-mechanical (QM) description of a $\left(\mathrm{H}_{2} \mathrm{O}\right)_{12}^{+}$cluster with a classical (MM) description of surrounding water molecules. The electronic structure was obtained at the Hartree-Fock level of theory employing Koopmans' theorem to obtain singly-ionized states and using the 6-31G basis set as implemented in our software package XMOLECULE $(26,27)$. (See SM for details).

Our simulations explored to what extent transient x-ray absorption is sensitive to ultrafast structural dynamics in water. The time-resolved x-ray absorption spectrum of the valence hole is shown in Fig. 3A for up to $100 \mathrm{fs}$ after the initial hole formation. While initially electronic states down to HOMO-6 were populated, the trajectories arrived at the cationic ground state within 25 fs (Fig. 3B), and non-Born-Oppenheimer effects were no longer present. This was accompanied by hole localization on a comparable timescale.

Proton transfer can be characterized by the distance between the charge and hole center. Directly after ionization, the charge and hole are overlapped at the $\mathrm{H}_{2} \mathrm{O}^{+}$; as proton transfer proceeds, the hole stays on the $\mathrm{OH}$ moiety, while the charge is carried away by the $\mathrm{H}_{3} \mathrm{O}^{+}(15)$. 
Fig. 3C shows that the charge-hole separation is correlated with the completion of the proton transfer. Within our model using bond order analysis (28), the proton transfer timescale was found to be $60 \mathrm{fs}$, in good agreement with Ref. (15). We further investigated the chemical environment surrounding the $\mathrm{H}_{2} \mathrm{O}^{+} / \mathrm{OH}$, where the valence hole is located, to extract correlations with $x$-ray spectral signatures. The average distance of the $\mathrm{H}_{2} \mathrm{O}^{+} / \mathrm{OH}$-oxygen to the nearest $\mathrm{H}_{2} \mathrm{O}$-oxygen is shown in Fig. 3D along with the spectral shift (the peak position of Fig. 3A). Initially, the partially negatively charged oxygens are pulled towards the region of positive charge $\left(\mathrm{H}_{2} \mathrm{O}^{+}\right)$, giving rise to the redshift of the absorption peak. Note that this motion initiates proton transfer, and thus at the point of maximum redshift, proton transfer is only about $25 \%$ complete (Fig. 3C). As a consequence of charge-hole separation, the distance between $\mathrm{OH}$ and the neighboring oxygens increases, and the redshift shrinks. In this way, proton transfer is indirectly reflected in the time evolution of the x-ray absorption peak position.

The results presented here offer new insight into the elementary dynamics of the highly reactive and short-lived ion and neutral radical species in ionized liquid water. Our experimental approach offers several advantages compared to previous attempts $(6,13,15)$ to determine the lifetime of the elusive $\mathrm{H}_{2} \mathrm{O}^{+}$radical cation. First, compared to traditional two-photon UV photoionization, strong-field ionization provides access to a large concentration of ionized species in solution. Assuming a cross section of $\sim 7$ Mbarn for the $\mathrm{O} 1$ s resonant absorption of the $\mathrm{OH}$ radical, we estimated an ionization fraction of $\sim 0.8 \%$ under our experimental conditions. Second, the $\mathrm{H}_{2} \mathrm{O}^{+}$and $\mathrm{OH}$ radicals exhibit strong $\mathrm{O} 1 s \rightarrow 2 p$-like resonant transitions in the soft $\mathrm{x}$-ray absorption spectra because they possess open $\mathrm{O} 2 \mathrm{p}$ sub-shells. These resonances occur in the background-free, near-edge spectral region, enabling detection down to few millimolar concentration. In comparison, previous attempts by spectrally resolved visible transient absorption spectroscopy to observe the $\mathrm{H}_{2} \mathrm{O}^{+}$radical cation via its predicted absorption signature at $2.3 \mathrm{eV}$ were unsuccessful due to its weak absorption being masked by the strong, broad hydrated elec- 
tron absorption feature, and, further complicated by cross-phase modulation artifacts $(6,13,15)$.

The present study revealed three distinct timescales in the early-time dynamics of ionized liquid water: $46 \pm 10 \mathrm{fs}, 0.18 \pm 0.02 \mathrm{ps}$, and $14.2 \pm 0.4 \mathrm{ps}$, tentatively assigned to the decay of the $\mathrm{H}_{2} \mathrm{O}^{+}$radical cation via proton transfer, vibrational cooling of the hot $\mathrm{OH}$ radical produced from $\mathrm{H}_{2} \mathrm{O}^{+}$, and geminate recombination of $\mathrm{OH}$ with the hydrated electron byproduct, respectively. The first two processes, hitherto unobserved, are of particular interest. The assignment of the 46-fs component to the lifetime of the $\mathrm{H}_{2} \mathrm{O}^{+}$cation is supported by QM/MM molecular dynamics simulations, which predict a lifetime of 60 fs. However, we note that the same simulations also showed that hole localization occurs within $30 \mathrm{fs}$. As such, with the presently available time resolution, hole localization also contributed to the observed dynamics. On the intermediate timescale, the 0.18 -ps component was tentatively assigned to vibrational cooling of hot $\mathrm{OH}$ radicals, produced upon ultrafast proton transfer. The formation of a vibrationally excited $\mathrm{OH}$ product from $\mathrm{H}_{2} \mathrm{O}^{+}$is likely considering the $3 \%$ change in equilibrium $\mathrm{OH}$ bond lengths between gas-phase $\mathrm{H}_{2} \mathrm{O}^{+}(0.9992 \AA)$ (29) and OH (0.9697 $\AA$ ) (30). The assignment of the 0.18-ps component to vibrational relaxation of the $\mathrm{OH}$ radical is supported by studies of the $\mathrm{OH}$ stretch of liquid $\mathrm{H}_{2} \mathrm{O}$ where numerous time-resolved infrared spectroscopic measurements show vibrational relaxation and vibrational energy transfer occurring within $0.2-0.3$ ps $(31,32)$.

We expect that a future study with higher time resolution and signal-to-noise ratios could furnish a more accurate $\mathrm{H}_{2} \mathrm{O}^{+}$lifetime and at the same time, permit the observation of ultrafast dynamics involving possible hole alignment, coherences and non-adiabatic dynamics induced by radiolysis. With improved statistics over a wide range of probe photon energies, we also anticipate the possibility of isolating the spectral signatures of $\mathrm{H}_{2} \mathrm{O}^{+}$and the hot $\mathrm{OH}$ radical. Interestingly, in addition to valence hole dynamics observed at $526 \mathrm{eV}$, the soft $\mathrm{x}$-ray probe appeared to be sensitive to the dynamics of the electron that was injected into the solvent by ionization. The spectral changes observed in the vicinity of the pre-edge absorption (531.0 
- $533.7 \mathrm{eV}$ ) fit a universal time constant of $0.26 \pm 0.03 \mathrm{ps}$ that matches previous studies of hydrated electron formation $(7,8)$ (See SM). Future experiments focusing on the pre- and mainedges of ionized liquid water which require a sub-micrometer-thick liquid jet could potentially yield the electronic energy level diagram of ionized liquid water (33) and shed light on its electronic relaxation dynamics, as well as possibly discern the cavity vs. non-cavity model for the structure of the hydrated electron (34).

\section{References}

1. B.C. Garrett et al., Chem. Rev. 105, 355-389 (2005).

2. S. Gordon in Early Developments in Radiation Chemistry, edited by J. Kroh (Royal Society of Chemistry (1989)) pp 163.

3. E. Alizadeh, L. Sanche, Chem. Rev. 112, 5578-5602 (2012).

4. F.E. Garrett-Bakelman et al., Science 364, 144 (2019).

5. M. Inokuti, Rev. Mod. Phys. 43, 297-347 (1971).

6. F. H. Long, H. Lu, K. B. Eisenthal, Phys. Rev. Lett. 64, 1469-1472 (1990).

7. A. Migus, Y. Gauduel, J. L. Martin, A. Antonetti, Phys. Rev. Lett. 58, 1559-1562 (1987).

8. P. Kambhampati, D.H. Son, T.W. Kee, P.F. Barbara, J. Phys. Chem. A 106, 2374-2378 (2002).

9. M.H. Elkins, H.L. Williams, A.T. Shreve, D.M. Neumark, Science 342, 1496-1499 (2013).

10. J. Li, Z. Nie, Y.Y. Zheng, S. Dong, Z.-H. Loh, J. Phys. Chem. Lett 4, 3698-3703 (2013).

11. J.M. Herbert, M.P. Coons, Annu. Rev. Phys. Chem 68, 447-472 (2017). 
12. E. J. Hart, J.W. Boag, J. Am. Chem. Soc. 84, 4090-4095 (1962).

13. Y. Gauduel, S. Pommeret, A. Migus, A. Antonetti, Chem. Phys. 149, 1-10 (1990).

14. E. Kamarchik, O. Kostko, J.M. Bowman, M. Ahmed, A.I. Krylov, J. Chem. Phys. 132, $194311(2010)$.

15. O. Marsalek et al., J. Chem. Phys. 135, 224510 (2011).

16. Th. Fransson et al., Chem. Rev. 116, 7551-7569 (2016).

17. C. Elles et al., J. Chem. Phys 130, 084501- (2009).

18. P. Emma et al., Nat. Photon. 4, 641-647 (2010).

19. B. Winter, E.F. Aziz, U. Hergenhahn, M. Faubel, I.V. Hertel, J. Chem. Phys. 126, 124504 (2007).

20. K. Nishizawa et al., Phys. Chem. Chem. Phys., 13, 413417 (2011).

21. S. Stranges, R. Richter, M. Alagia, J. Chem. Phys. 116, 3676-3680 (2002).

22. R.A. Crowell, D.M. Bartels, J. Phys. Chem. 100, 17940-17949 (1996).

23. J.H. Guo et al., Phys. Rev. Lett. 89, 137402 (2002).

24. M.J. Rosker, M. Dantus, A.H. Zewail, J. Chem. Phys. 89, 6113-6127 (1988).

25. J.C. Tully, J. Chem. Phys. 93, 1061-1071 (1990).

26. Y. Hao, L. Inhester, K. Hanasaki, S.-K. Son, R. Santra, Struct. Dyn. 2, 041707 (2015).

27. K. Khalili, L. Inhester, C. Arnold, R. Welsch, J. W. Andreasen, R. Santra, Struct. Dyn. 6, 044102 (2019). 
28. L. Inhester et al., J. Phys. Chem. Lett. 9, 1156-1163 (2018).

29. T.R. Huet, C.J. Pursell, W.C. Ho, B.M. Dinelli, T. Oka, J. Chem. Phys. 97, 5977-5987 (1992).

30. K.P. Huber, G. Herzberg in NIST Chemistry WebBook, NIST Standard Reference Database Number 69 (Eds P. J. Linstrom and W. G. Mallard) (National Institute of Standards and Technology) Available at http://webbook.nist.gov.

31. F. Perakis, L. De Marco, A. Shalit, F. Tang, Z. R. Kann, T.D. Khne, R. Torre, M. Bonn, Y. Nagata, Chem. Rev. 116, 7590-7607 (2016).

32. M.L. Cowan, B.D. Bruner, N. Huse, J.R. Dwyer, B. Chugh, E.T.J. Nibbering, T. Elsaesser, R.J.D. Miller, Nature 434, 199-202 (2005).

33. A.P. Gaiduk, T.A. Pham, M. Govoni, F. Paesani, G. Galli, Nat. Commun. 9, 247 (2018).

34. R.E. Larsen, W.J. Glover, B.J. Schwartz, Science 329, 65-69 (2010).

35. M.L. Vidal, X. Feng, E. Epifanovsky, A.I. Krylov, S. Coriani, J. Chem. Theo. Comp. 15, 3117-3133 (2019).

36. M. Nagasaka, T. Hatsui, T. Horigome, Y. Hamamura, N. Kosugi, J. Elec. Spec. Rel. Pheno. 177, 130-134 (2010).

37. L. Young, Z.-H. Loh, R. Santra, C. Arnold, Data for Observation of the fastest chemical processes in the radiolysis of water. Zenodo repository. DOI: $10.5281 /$ zenodo. 3543680

38. W. F. Schlotter et al., Rev. Sci. Instrum. 83, 043107 (2012).

39. J. Koralek et al., Nat. Commum. 9, 1353 (2019). 
40. P. Heimann et al., Rev. Sci. Instrum. 82, 093104 (2011).

41. Y-D. Chuang et al., Rev. Sci. Instrum. 88, 013110 (2017).

42. D.J. Higley et al., Rev. Sci. Instrum. 87, 033110 (2016).

43. M. Beye et al., Appl. Phys. Lett. 100, 121108 (2012).

44. J. Niskanen, M. Fondella, C. J. Sahle, S. Eckert, R. M. Jay, K. Gilmore, A. Pietzsch, M. Dantze, X. Lue, D. E. McNally, T. Schmitt, V. Vaz da Cruz, V. Kimberg, F. Gel'mukhanov, and A. Föhlisch, Proc. Nat. Acad. Sci. 116, 4058 - 4063 (2019).

45. K. Yamazoe, J. Miyawaki, H. Niwa, A. Nilsson, Y. Harada, Measurements of Ultrafast Dissociation in Resonant Inelastic X-ray Scattering of Water, J. Chem. Phys. 150, 204201 (2019).

46. C.D. Cappa, J.D. Smith, B.M. Messer, R.C. Cohen, R.J. Saykally, J. Phys. Chem. A 111, 4776-4785 (2007).

47. D. Prendergast, G. Galli, Phys. Rev. Lett. 96, 215502 (2006).

48. H. Wen, N. Huse, R. W. Schoenlein, A. M. Lindenberg, J. Chem. Phys. 131, 234505 (2009).

49. F. Maseras and K. Morokuma, J. Comp. Chem. 16, 11701179, (1995).

50. P. Mark and L. Nilsson, J. Phys. Chem. A 105, 9954-9960 (2001).

51. H.C. Andersen, J. Comp. Phys. 52, 24-34 (1983).

\section{Acknowledgments}

Funding: This work was supported by the U.S. Department of Energy, Office of Science, Basic Energy Science, Chemical Sciences, Geosciences and Biosciences Division who supported 
the Argonne group under contract number DE-AC02-06CH11357. Use of the Linac Coherent Light Source (LCLS), SLAC National Accelerator Laboratory, and, resources of the Center for Nanoscale Materials (CNM), Argonne National Laboratory, are supported by the U.S. Department of Energy (DOE), Office of Science, Office of Basic Energy Sciences (BES) under Contracts DE-AC02-76SF00515 and DE-AC02-06CH11357. L.Y. acknowledges support from Laboratory Directed Research and Development (LDRD) funding from Argonne National Laboratory for conceptual design and proposal preparation. Z.-H.L., T.D., and M.S.B.M.Y. acknowledge support from the Singapore Ministry of Education (MOE2014-T2-2-052 and RG105/17). L.K., J.-E.R. acknowledge support from the Swedish Science Council (2018-04088). M.S. was supported by the CNRS GotoXFEL program. C.A., R.W., and R.S. were supported by the Cluster of Excellence 'Advanced Imaging of Matter' of the Deutsche Forschungsgemeinschaft (DFG) - EXC 2056 - project ID 390715994. L.I. and R.S. acknowledge support by the Chemical Sciences, Geosciences, and Biosciences Division, Office of Basic Energy Sciences, Office of Science, U.S. Department of Energy, Grant No. DE-SC0019451. K.K. was supported by the European Research Council (ERC) under the European Union's Horizon 2020 research and innovation programme (Grant Agreement No. 681881). A.I.K. and K.N. were supported by the U.S. National Science Foundation (No. CHE-1856342). A.I.K. is also a grateful recipient of the Simons Fellowship in Theoretical Physics and Mildred Dresselhaus Award from the Hamburg Centre for Ultrafast Imaging, which supported her sabbatical stay in Germany. Author contributions: L.Y., Z.-H.L. and R.S. originated the project concept. W.F.S., G.D., M.-F.T., R.D.S. and S.H.S. designed the experimental apparatus and characterized the target. Z.-H.L., G.D., C.A., L.K., S.H.S., A.A.H., Y.K., M.-F.T., P.J.H., A.M.M., M.S.B.M.Y., T.D. M.S., S.M, G.C., J.K., M.P.M., W.F.S., J.-E.R., and L.Y. executed the experiment and collected the experimental data. Z.-H.L., Y.K., L.K., J.-E.R. and L.Y. analyzed the experimental data. R.S., C.A., R.W., L.I, K.K., K.N. and A.K. provided supporting theoretical calculations. L.Y., Z.-H.L., R.S. and 
C.A. wrote the paper with comments from all authors. L.Y. was responsible for the coordination of the project. Competing interests: A.I.K. is a board member and a part-time owner of Q-Chem. Inc.. Data and materials availability: The underlying data are available in the supplementary materials and Zenodo, the CERN repository (37).

\section{Supplementary materials}

Materials and Methods

Supplementary Text

Figs. S1 to S11

Tables S1 to S3

References (38-51) 
Fig. 1. Ultrafast $x$-ray laser probe of ionization dynamics of liquid water. (A) Ionization of $\mathrm{H}_{2} \mathrm{O}$ produces $\mathrm{H}_{2} \mathrm{O}^{+}$with a strong isolated soft x-ray absorption resonance $\left(1 \mathrm{a}_{1} \rightarrow 1 \mathrm{~b}_{1}\right)$. (B) Absorption resonances in the gas phase for the four species associated with elementary proton transfer: $\mathrm{H}_{2} \mathrm{O}^{+}+\mathrm{H}_{2} \mathrm{O} \rightarrow \mathrm{OH}+\mathrm{H}_{3} \mathrm{O}^{+}$calculated with 6-311(2+,+) $\mathrm{G}(2 \mathrm{df}, \mathrm{p})$ basis with uncontracted core (oxygen) using fc-cvs-EOM-CCSD (35). Only $\mathrm{H}_{2} \mathrm{O}^{+}$and $\mathrm{OH}$ fall outside the region of strong absorption by liquid water (36), i.e. in the water window. (C) Schematic of the experimental setup: 800-nm strong-field ionization pump and time-delayed $\mathrm{x}$-ray probe. The x-ray probe is monitored simultaneously in transmission, total fluorescence and dispersed fluorescence as a function of pump-probe time delay and incident photon energy. See Materials and Methods.

Fig. 2. Transient $x$-ray absorption spectroscopy for the reaction $\mathrm{H}_{2} \mathrm{O}^{+}+\mathrm{H}_{2} \mathrm{O} \rightarrow \mathrm{OH}$ $+\mathbf{H}_{3} \mathbf{O}^{+}$. (A) Absorption for $\Delta t<0$ and $\Delta t>100$ fs monitored through the dispersed fluorescence channel. (B) Differential absorption $\Delta A$ in the valence hole $\left(\mathrm{H}_{2} \mathrm{O}^{+} / \mathrm{OH}\right)$ region. Spectra collected at 216 time delays between 1.55 .8 ps are averaged to produce the resonance profile, which is fit to a sum of two Lorentzians. Error bars correspond to the standard deviation. A total of 31 energy sweeps and 27546 shots are collected. (C) X-ray absorption spectrum of the aqueous $\mathrm{OH}$ radical. (D) Polarization averaged time traces at three $\mathrm{x}$-ray probe energies, $525.43,525.93$ and $526.73 \mathrm{eV}$.

Fig. 3. Calculated x-ray absorption spectra and trajectory analysis. (A) Resonant x-ray absorption spectrum of ionized water. (B) Hole radius (green) and non-adiabatic dynamics (gray, solid line shows average). (C) Charge-hole distance (CHD) (blue) and completed proton transfer percentage (orange). (D) Average distance between oxygens in $\mathrm{H}_{2} \mathrm{O}$ molecules (black) along with the average distance of the $\mathrm{H}_{2} \mathrm{O}^{+} / \mathrm{OH}$ oxygen to the nearest oxygen (purple) and the spectral shift of the $\mathrm{x}$-ray absorption resonance (red). 


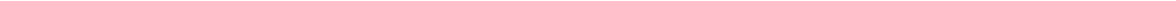




\section{Supplementary Information: Observation of the fastest chemical processes in the radiolysis of water}

Z.-H. Loh, ${ }^{1 *}$ G. Doumy, ${ }^{2}$ C. Arnold, ${ }^{3,4,5}$ L. Kjellsson, ${ }^{6,7}$ S.H. Southworth, ${ }^{2}$ A. Al Haddad, ${ }^{2}$ Y. Kumagai, ${ }^{2}$ M.-F. Tu, ${ }^{2}$ P.J. Ho, ${ }^{2}$, A.M. March, ${ }^{2}$ R.D. Schaller, ${ }^{8,9}$ M.S. Bin Mohd Yusof, ${ }^{1}$ T. Debnath, ${ }^{1}$ M. Simon, ${ }^{10}$ R. Welsch, ${ }^{3,5}$ L. Inhester, ${ }^{3}$ K. Khalili, ${ }^{11}$ K. Nanda,${ }^{12}$ A.I. Krylov, ${ }^{12}$ S. Moeller ${ }^{13}$ G. Coslovich, ${ }^{13}$ J. Koralek, ${ }^{13}$ M.P. Minitti, ${ }^{13}$ W.F. Schlotter ${ }^{13}$ J.-E. Rubensson, ${ }^{6}$, R. Santra, ${ }^{3,4,5 *}$ L. Young ${ }^{2,14 *}$

${ }^{1}$ Division of Chemistry and Biological Chemistry, School of Physical and Mathematical Sciences,

Nanyang Technological University, Singapore

${ }^{2}$ Chemical Sciences and Engineering Division, Argonne National Laboratory, Lemont, IL USA

${ }^{3}$ Center for Free-Electron Laser Science, DESY, Hamburg, Germany

${ }^{4}$ Department of Physics, Universität Hamburg, Hamburg, Germany

${ }^{5}$ The Hamburg Centre for Ultrafast Imaging, Hamburg, Germany

${ }^{6}$ Department of Physics and Astronomy, Uppsala University, Uppsala, Sweden

${ }^{7}$ European XFEL GmbH, Schenefeld, Germany

${ }^{9}$ Center for Nanoscale Materials, Argonne National Laboratory, Lemont, IL, USA

${ }^{10}$ Department of Chemistry, Northwestern University, Evanston, IL, USA

${ }^{11}$ Sorbonne Université and CNRS, Laboratoire de

Chemie Physique-Matière et Rayonnement, LCPMR, F-750005, Paris, France

${ }^{12}$ Department of Energy Conversion and Storage,

Technical University of Denmark, Roskilde, Denmark

${ }^{13}$ Department of Chemistry, University of Southern California, Los Angeles, CA, USA

${ }^{14}$ LCLS, SLAC National Accelerator Laboratory, Menlo Park, CA, USA

${ }^{15}$ Department of Physics and James Franck Institute, The University of Chicago, Chicago, IL, USA

*To whom correspondence should be addressed;

E-mail: zhiheng@ntu.edu.sg, robin.santra@cfel.de, young@anl.gov 


\section{Methods and Materials}

The optical ionization pump-soft x-ray probe measurements were performed at the soft x-ray (SXR) endstation (38) of the Linac Coherent Light Source (LCLS) x-ray free-electron laser (XFEL). The 800-nm output of a Ti:sapphire amplifier is focused onto a 2 - $\mu \mathrm{m}$-thick colliding liquid sheet jet target (39) to ionize liquid water by strong-field ionization. The surface of the liquid jet is oriented at $45^{\circ}$ relative to the incident $800-\mathrm{nm}$ ionization pump and soft $\mathrm{x}$-ray probe beams, with a crossing angle of $\sim 1^{\circ}$ between the two beams. With a pulse energy of 1.1 $\mathrm{mJ}$, a focal spot size $\left(1 / \mathrm{e}^{2}\right.$ diameter $)$ of $170(\mathrm{~h}) \times 120(\mathrm{v}) \mu \mathrm{m}^{2}$, and a pulse duration of $60 \mathrm{fs}$, the peak intensity of the ionization pump pulse is $2.3 \times 10^{14} \mathrm{~W} / \mathrm{cm}^{2}$, approximately one order of magnitude higher than that used in an earlier study of strong-field-ionized liquid water by ultrafast optical spectroscopy (10). The 20-fs XFEL pulse (40 fs for the survey scan shown in Fig. 2A), is monochromatized to a spectral width of $0.1 \mathrm{eV}$. This process also stretches the 20-fs pulse (40) to $\sim 30$ fs. The monochromatized XFEL pulse is then focused by a pair of Kirkpatrick-Baez mirrors to a focal spot size $\left(1 / \mathrm{e}^{2}\right.$ diameter) of $108(\mathrm{~h}) \times 17(\mathrm{v}) \mu \mathrm{m}^{2}$ on the ionized water target. The intensity and polarization of the 800-nm pump laser can be controlled by a $\lambda / 2$, polarizer, $\lambda / 2$ combination in the $800-n m$ delivery path.

Three different observables are recorded simultaneously in the experiments as shown in

Fig. S1. The transmitted x-ray pulse energy is recorded by an x-ray CCD (upper panel); the spectrally-integrated $\mathrm{x}$-ray fluorescence is monitored by a pair of silicon avalanche photodiodes (3-mm diameter, SAR3000G1 Laser Components) placed $\sim 3.2 \mathrm{~cm}$ from the target for a solid angle of $5.5 \times 10^{-4}$ (middle panel); and the spectrally-resolved $\mathrm{x}$-ray fluorescence is recorded by an $\mathrm{x}$-ray emission spectrometer, with a collection/transmission efficiency of $\sim 10^{-7}$ (41) (lower panel). The transmission channel has the superior signal-to-noise ratio and was used for the kinetics analyses. 


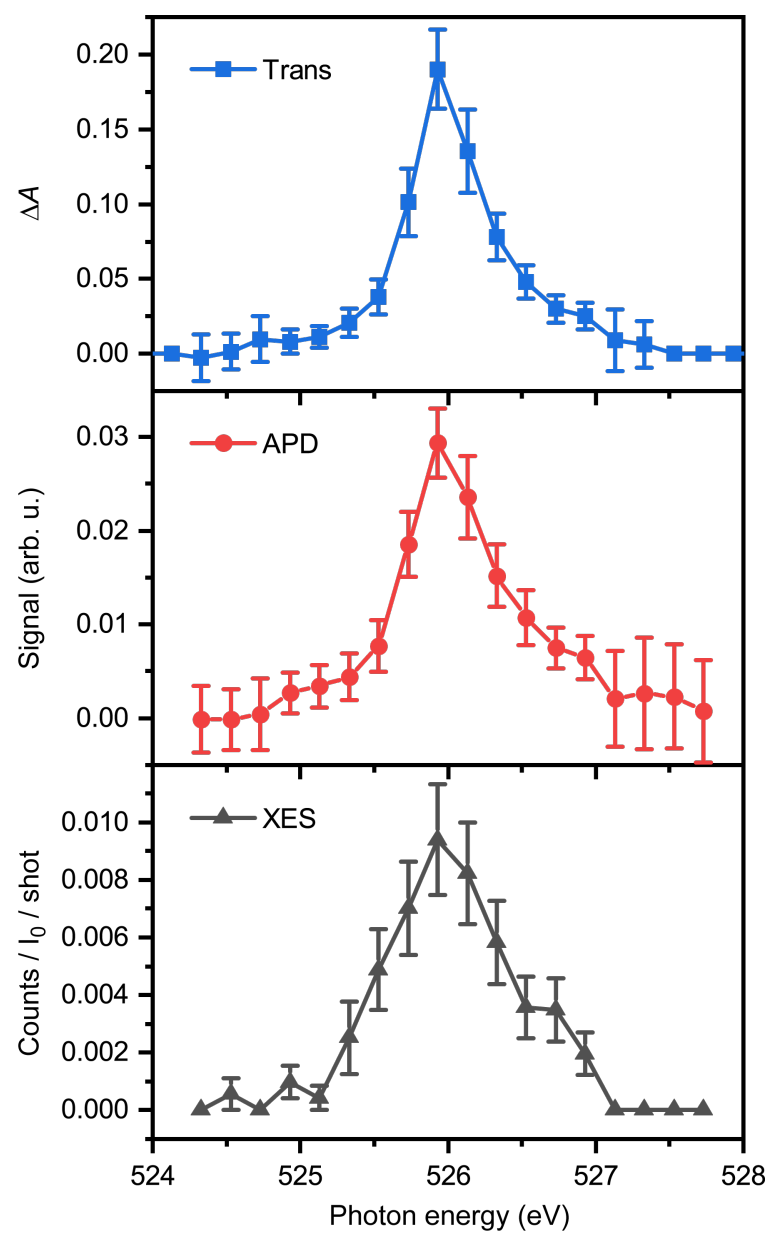

Figure S1: Simultaneous detection channels for x-ray transient absorption. Survey scan from 524-528 eV with 0.1-eV bandwidth, 30-fs pulses averaged over delay times 1-6 ps. A total of 31,181 out of 158,680 shots met the joint criteria for linear detector response to FEL intensity, acceptable time jitter, and laser power. Upper panel: transmission channel (differential absorption). Middle panel: APD signal (total fluorescence yield). Lower panel: dispersed emission (partial fluorescence yield). There are 24 counts at the maximum, a total of 130 counts and error bars are statistical.

Upstream of the liquid-jet endstation, a gas detector records the pulse energy of every XFEL pulse in the front-end enclosure for single-shot referencing and rejection of spurious pulses and a monochromator scans the incident x-ray photon energy in the range of $518-538 \mathrm{eV}$. The incident fluence monitor, $I_{0}$, of the monochromatized x-ray beam was checked for linearity with 
respect to the upstream gas monitor; higher energy pulses in the nonlinear region were rejected from the transmission data analysis (42). An arrival time monitor positioned in the upstream Hutch 1 endstation monitors the relative arrival times of the optical laser and the XFEL pulses. In these experiments, the optical-XFEL timing jitter limits the effective time resolution to $\sim 110$ fs (43).

The monochromator energy calibration (shift and dispersion) was determined as follows. The energy shift relative to the monochromator readout was determined by comparing the observed rise of the pre-edge absorption in our $2-\mu \mathrm{m}$ water jet with that in a $800-\mathrm{nm}$ water jet by Nagasaka et al. (36). The thickness of our colliding water jet at a flow rate of $3.5 \mathrm{ml} / \mathrm{min}$ (linear flow rate $\approx 40 \mathrm{~m} / \mathrm{s}$ ) was measured using white light interference fringes to be $2.09 \pm 0.10 \mu \mathrm{m}$ at the Center for Nanoscale Materials at Argonne National Laboratory. This value was used to determine the thickness of the jet in the experimental configuration, i.e. at $45^{\circ}$ incidence angle, and to scale the absorbance values of Ref. (36). Using jet-on/jet-off data for 5 separate runs, we determined an energy shift relative to the monochromator readout of $-2.07 \pm 0.07 \mathrm{eV}$ using the inflection of the pre-edge resonance as a reference point. The dispersion of the monochromator was checked with two reference points, the pre-edge feature as described above and the water XES emission doublet from the $1 b_{1}$ orbital collected in the dispersed fluorescence channel for incident energies between 536 - $538 \mathrm{eV}$ (44). In a single scan from 518 - $538 \mathrm{eV}$, we observe both reference points. The RIXS spectrum of the $\mathrm{OH}$ radical appears at the $\mathrm{OH}$ resonance energy $(526 \mathrm{eV})$ and its elastic peak coincides with the lower energy partner of the water XES doublet. We find that the calibrated dispersion of the monochromator does not introduce an error larger than $0.07 \mathrm{eV}$ (better than 1\%) and use it directly. (We note that by using an alternative reported position for the XES emission doublet (45) would increase the monochromator dispersion by $5 \%$, and shift the $\mathrm{OH}$ radical resonance by $-0.4 \mathrm{eV}$.) 


\section{Intensity Dependence}

Measurements were performed to investigate the dependence of the early-time dynamics on the intensity of the strong-field ionization pump pulse. In these measurements, the pump power was varied by using a combination of a half-wave plate and a linear polarizer while keeping the pulse duration and focal spot sizes fixed. The differential absorption signal at $525.93 \mathrm{eV}$, near the peak of the $\mathrm{OH}$ radical absorption, was recorded as a function of time delay for various fractions of the full pump intensity $\left(2.3 \times 10^{14} \mathrm{~W} / \mathrm{cm}^{2}\right)$. All the time traces can be fit to a rise time of $49 \pm 7$ fs (Fig. S2), consistent with the time constant of $46 \pm 10$ fs obtained from the global analysis of the time traces shown in Fig. 2D of the main text. The absence of any intensity dependence suggests that the observed dynamics arise from the chemical dynamics of ionized liquid water rather than the collective, macroscopic dynamics of a plasma generated by strong-field ionization. It is noteworthy that the $\Delta A$ value in the positive-time region is only 0.007 for the lowest peak intensity $\left(9.5 \times 10^{13} \mathrm{~W} / \mathrm{cm}^{2}\right)$ employed in the intensity-dependent measurements.

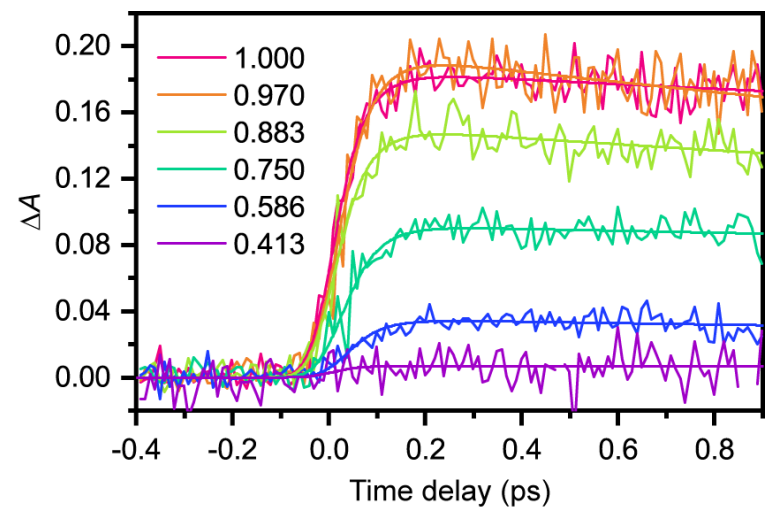

Figure S2: Intensity dependence of early time kinetics at different fractions of the full pump intensity. Differential absorption traces for an incident photon energy of $526 \mathrm{eV}$. 


\section{Sequential Kinetics Modeling}

Surface Fit (Fig. 2B) A sequential kinetics model is used to fit the time-resolved $\Delta A$ spectrum taken in the $2 \mathrm{D}$ survey scan shown in Figure 2B. The model assumes that the species initially produced by ionization decays with lifetime $\tau_{1}$ to give an intermediate species with lifetime $\tau_{2}$, and that the absorption spectrum associated with each species is $S_{1}(E)$ and $S_{2}(E)$, where $E$ is the probe photon energy. Within this framework, the $\Delta A$ signal as a function of time delay $\tau$ and $E$ is given by the expression

$$
\Delta A(E, t)=\left[S_{1}(E) e^{-t / \tau_{1}}+\frac{S_{2}(E) \tau_{2}}{\tau_{2}-\tau_{1}}\left(e^{-t / \tau_{2}}-e^{-t / \tau_{1}}\right)\right] H(t) * \operatorname{IRF}(t)
$$

where $H(t)$ is the Heaviside function. In our analysis we assume that $\tau_{1} \ll 1$ ps, as suggested by the observed rapid narrowing of the $\Delta A$ spectrum on the sub-picosecond timescale, hence allowing the absorption lineshape $S_{2}(E)$ to be obtained by averaging the differential absorption spectra collected at pump-probe time delays $>1.5 \mathrm{ps}$.

Global Fit (Fig. 2D) Fig. 2D shows a clearly delayed appearance of the isotropic $\Delta A$ signal at $525.93 \mathrm{eV}$ relative to 525.43 and $526.73 \mathrm{eV}$. The improved signal-to-noise ratio relative to the $2 \mathrm{D}$ data in Fig. $2 \mathrm{~B}$ is due to a 10 -fold increase in the number of shots per time bin.

The three time traces, prior to normalization, are fit to the function

$$
\Delta A(t)=I R F(t) * H(t) \sum_{i=1}^{n} A_{i} e^{-t / \tau_{i}}
$$

where $n$ specifies the number of exponential components, each with amplitude $A_{i}$ and time constant $\tau_{i}$. Each component is multiplied with the Heaviside function $H(t)$ before the product is convolved with the instrument response function $\operatorname{IRF}(t)$. The full-width at half-maximum (FWHM) of $I R F(t)$, the time-zero value $\left(t_{0}\right)$, and the time constants are set as shared parameters in the global fit. 
In the spirit of the surface fit, we first attempted a fit to two time constants, with fixed $t_{0}$ and IRF. We find that it cannot reproduce the observed ordering of the delayed rise, which experimentally is $525.43 \mathrm{eV}<526.73 \mathrm{eV}<525.93 \mathrm{eV}$ as displayed in Fig. S3. That is, the red-shifted trace rises first, followed by the blue-shifted trace and finally by the central trace (black). The best fit, however, shows the blue trace to rise first, rather than the red trace.

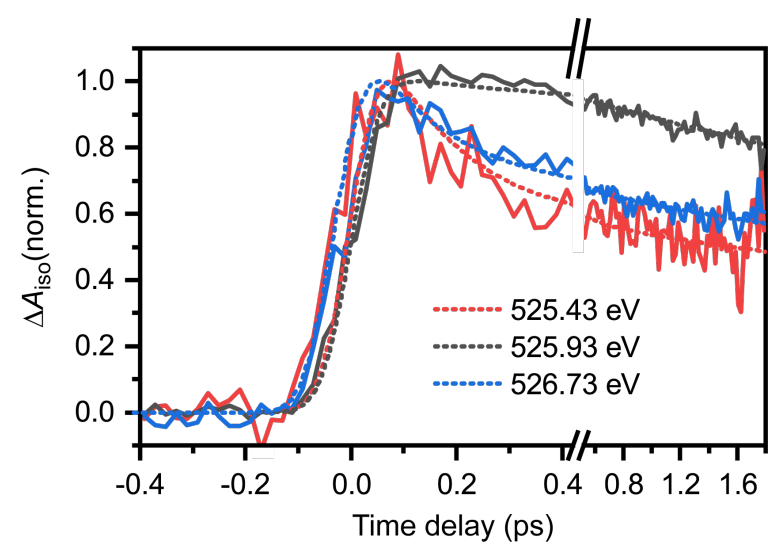

Figure S3: $\Delta A_{\text {iso }}$ traces (same as Fig 2D main text) for three different incident photon energies shown with the best fit using two-time constants.

Next we allowed the width of the instrument response function and the time-zero position to vary across the three probe photon energies while globally fitting the time traces to only two decay components. The resultant time-zero shifts $\left(t_{0}\right)$ and full-width at half-maxima (FWHM) of the instrument response functions are summarized in Table S2 along with the time constants and amplitudes.

It is evident that significant deviations in the $t_{0}$ and FWHM values are needed to fit the time traces. In particular, in order to reproduce the pronounced delay in the rise of the signal at $525.93 \mathrm{eV}$, the FWHM value of the instrument response has to increase by $20 \%$ and the timezero position shifts towards positive time by $44 \mathrm{fs}$. In addition to these significant changes in the $t_{0}$ and FWHM values, we also note the non-monotonic trend in the $\Delta t_{0}$ values as a function of X-ray photon energy. These observations are unrealistic, given that the monochromator is 
Table S1: Parameters obtained from fitting the time traces in Figure S4 to independent $I R F(t)$, FWHM and time-zero values.

\begin{tabular}{cccc}
\hline \hline & $525.43 \mathrm{eV}$ & $525.93 \mathrm{eV}$ & $526.73 \mathrm{eV}$ \\
\hline$A_{1}$ & $0.0174 \pm 0.0036$ & $0.0286 \pm 0.0052$ & $0.0367 \pm 0.0042$ \\
$A_{2}$ & $0.0249 \pm 0.0014$ & $0.2295 \pm 0.0054$ & $0.0613 \pm 0.0020$ \\
$\tau_{1}(\mathrm{ps})$ & & $0.19 \pm 0.03$ & \\
$\tau_{2}(\mathrm{ps})$ & & $8.2 \pm 1.1$ & \\
$\Delta t_{0}$ & $-23 \pm 57$ & $44 \pm 24$ & $19 \pm 33$ \\
$\mathrm{FWHM}(\mathrm{fs})$ & $113 \pm 24$ & $142 \pm 4$ & $133 \pm 12$ \\
\hline \hline
\end{tabular}

tuned by only -0.5 and $+0.8 \mathrm{eV}$ from a central energy of $525.93 \mathrm{eV}$.

The addition of a third time component allows us to fit the early time dynamics as shown in Fig. S4 using the values summarized in Table S2

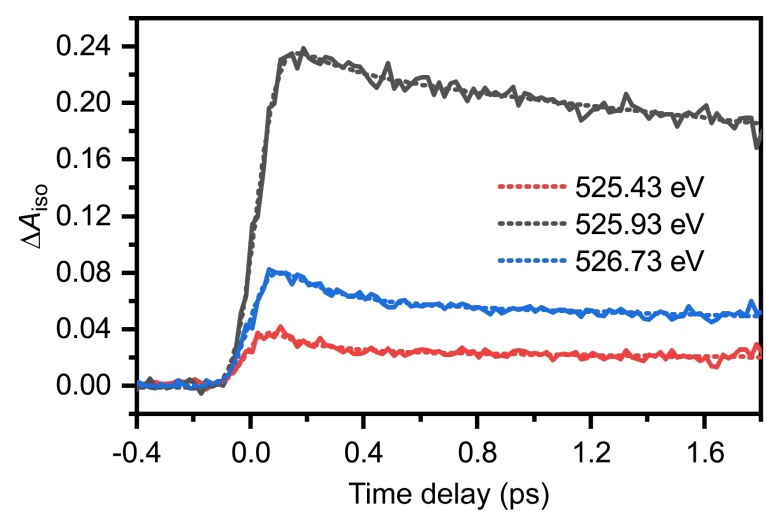

Figure S4: Unnormalized $\Delta A_{\text {iso }}$ traces (same as Fig 2D main text) for three different incident photon energies. The fit to a three exponential component function is also shown.

The new 46-fs component $\left(A_{3}\right)$ is, within experimental error, absent at $525.43 \mathrm{eV}$, whereas it manifests itself as a rising signal at $525.93 \mathrm{eV}\left(A_{3}<0\right)$ and to a smaller extent at 526.73 $\mathrm{eV}$. The presence of the rising component accounts for the delayed appearance of the signal at 525.93 and $526.73 \mathrm{eV}$ relative to $525.43 \mathrm{eV}$. Both the 0.16-ps and 9.2-ps components appear as a decaying signal across all three probe photon energies. 
Table S2: Parameters obtained from fitting the time traces in Figure S4 to Eq. S2

\begin{tabular}{cccc}
\hline \hline & $525.43 \mathrm{eV}$ & $525.93 \mathrm{eV}$ & $526.73 \mathrm{eV}$ \\
\hline$A_{1}$ & $0.0238 \pm 0.0076$ & $0.0670 \pm 0.0270$ & $0.0569 \pm 0.0162$ \\
$A_{2}$ & $0.0242 \pm 0.0012$ & $0.2270 \pm 0.0053$ & $0.0597 \pm 0.0018$ \\
$A_{3}$ & $0.0008 \pm 0.0152$ & $-0.2907 \pm 0.0268$ & $-0.0623 \pm 0.0231$ \\
$\tau_{1}(\mathrm{ps})$ & & $0.16 \pm 0.03$ & \\
$\tau_{2}(\mathrm{ps})$ & & $9.2 \pm 1.3$ & \\
$\tau_{3}(\mathrm{fs})$ & & $46 \pm 10$ & \\
$t_{0}$ & & 0 & \\
FWHM $(\mathrm{fs})$ & & $119 \pm 7$ & \\
\hline \hline
\end{tabular}

The presence of three time constants leads us to consider sequential kinetics involving four species

$$
\mathrm{A} \stackrel{k_{A}}{\longrightarrow} \mathrm{B} \stackrel{k_{B}}{\longrightarrow} \mathrm{C} \stackrel{k_{C}}{\longrightarrow} \mathrm{D}
$$

with rate constants $k_{A}, k_{B}$, and $k_{C}$. From the main text, A, B, C, and D correspond to $\mathrm{H}_{2} \mathrm{O}^{+}$, the vibrationally excited $\mathrm{OH}$ radical $\left(\mathrm{OH}^{*}\right)$, the $\mathrm{OH}$ radical in the vibrational ground state, and the $\mathrm{OH}^{-}$anion. The time-dependent populations of these species are given by

$$
\begin{gathered}
N_{A}(t)=N_{0} e^{-k_{A} t} \\
N_{B}(t)=\frac{N_{0} k_{A}}{k_{B}-k_{A}}\left(e^{-k_{A} t}-e^{-k_{B} t}\right) \\
N_{C}(t)=\frac{N_{0} k_{A} k_{B}}{k_{B}-k_{A}}\left[\frac{1}{k_{C}-k_{A}}\left(e^{-k_{A} t}-e^{-k_{C} t}\right)-\frac{1}{k_{C}-k_{B}}\left(e^{-k_{B} t}-e^{-k_{C} t}\right)\right] \\
N_{D}(t)=N_{0}-\frac{N_{0} k_{B} k_{C}}{\left(k_{A}-k_{B}\right)\left(k_{A}-k_{C}\right)} e^{-k_{A} t}-\frac{N_{0} k_{A} k_{B}}{\left(k_{A}-k_{B}\right)\left(k_{B}-k_{C}\right)} e^{-k_{B} t} \\
-\frac{N_{0} k_{A} k_{B}}{\left(k_{A}-k_{C}\right)\left(k_{B}-k_{C}\right)} e^{-k_{C} t}
\end{gathered}
$$

In the above equations, $N_{0}$ is the initial population of species $\mathrm{A}$ that is created by strongfield ionization. Fig. S5 shows the temporal evolutions of the various populations, computed 
based on the rate constants given in Table $\mathrm{S} 1$ and after convolving with an instrument response function of 119 -fs FWHM. Note that the observed $\Delta A_{\text {iso }}$ time trace at a specific x-ray probe photon energy $E$ is related to the populations via

$$
\Delta A_{i s o}(E, t) \propto I R F(t) * H(t) \sum_{i=1}^{4} S_{i}(E) N_{i}(t)
$$

where $S_{i}(E)$ is the absorption spectrum of component $i$ and $N_{i}(t)$ is the time-dependent population of component $i$. In the $526-\mathrm{eV}$ region, only $\mathrm{H}_{2} \mathrm{O}^{+}, \mathrm{OH}^{*}$, and $\mathrm{OH}$ are expected to contribute to the $\Delta A_{\text {iso }}$ signal. The $\mathrm{OH}^{-}$absorption appears just to the red of the pre-edge feature around $534 \mathrm{eV}(46)$.

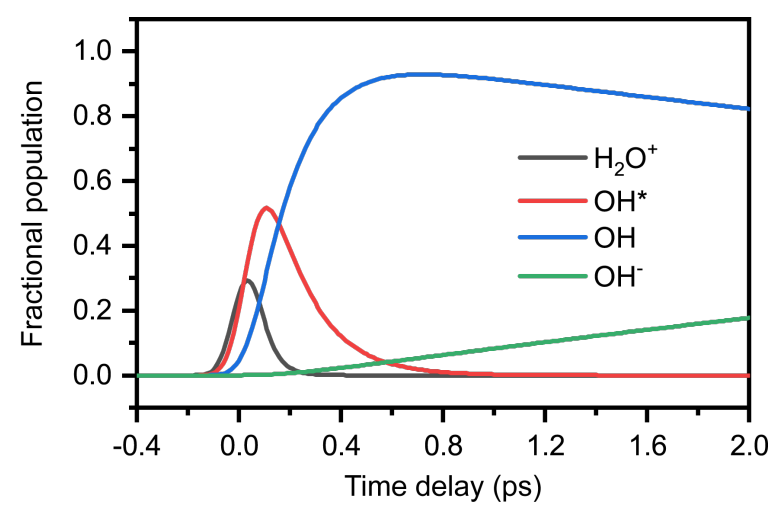

Figure S5: Sequential kinetics for $\mathrm{H}_{2} \mathrm{O}^{+} \rightarrow \mathrm{OH}^{*} \rightarrow \mathrm{OH} \rightarrow \mathrm{OH}^{-}$. $\tau_{1}=46$ fs, $\tau_{2}=0.16 \mathrm{ps}, \tau_{3}=9.2 \mathrm{ps}$. 


\section{Franck-Condon analysis of the $\mathrm{OH}$ absorption lineshape}

The absorption spectrum of the $\mathrm{OH}$ radical (Fig. 2C) shows a sideband that is associated with the vibrational progression of the $\mathrm{OH}$ stretch, i.e., the main peak corresponds to the $v^{\prime \prime}=0 \rightarrow v^{\prime}=$ 0 transition whereas the vibrational sideband corresponds to the $v^{\prime \prime}=0 \rightarrow v^{\prime}=1$ transition. The amplitude of the sideband is $(0.139 \pm 0.028) \times$ that of the main peak, which implies a Huang-Rhys factor $S$ of 0.139 . (The Huang-Rhys factor characterizes the displacement between the potential energy curves of the electronic ground state and the $\mathrm{O} 1 \mathrm{~s}^{-1}$ core-excited state.) Due to the difference in the $\mathrm{OH}$ bond length of $\mathrm{H}_{2} \mathrm{O}^{+}$and $\mathrm{OH}$, we expect the $\mathrm{OH}$ radical product to be initially formed in a vibrationally excited state. The Franck-Condon factors for transitions from the vibrationally excited $v^{\prime \prime}=1$ and $v^{\prime \prime}=2$ states of $\mathrm{OH}$ are summarized in Table S3; transitions from the $v^{\prime \prime}=0$ ground state are also included for comparison.

Based on the Franck-Condon factors, we compute the absorption lineshapes for the $\mathrm{OH}$ radical in the $v^{\prime \prime}=0,1$, and 2 states (Fig. S6). The results obtained from the fit in Fig. 2C give the energy spacing between adjacent transitions of $0.48 \mathrm{eV}$ and FWHM widths of each transition of $0.48 \mathrm{eV}$. Note that the absorption lineshapes for the vibrationally excited states of $\mathrm{OH}$ are significantly broader than that for the $v^{\prime \prime}=0$ ground state. Therefore, it is conceivable that the broader lineshape that is observed at early time delays in Fig. 2B arises from vibrationally hot $\mathrm{OH}$. For comparison, we also include in Fig. S6 the lineshape of $S_{1}(E)$, modelled by a Lorentzian with a FWHM width of $0.98 \mathrm{eV}$. 
Table S3: Franck-Condon factors for the various $v^{\prime \prime} \rightarrow v^{\prime}$ transitions of the $\mathrm{OH}$ radical.

\begin{tabular}{llll}
\hline \hline & $v^{\prime \prime}=0$ & $v^{\prime \prime}=1$ & $v^{\prime \prime}=2$ \\
\hline$v^{\prime}=0$ & 0.8700 & 0.1879 & 0.0084 \\
$v^{\prime}=1$ & 0.1211 & 0.6446 & 0.2097 \\
$v^{\prime}=2$ & 0.0084 & 0.3254 & 0.4651 \\
$v^{\prime}=3$ & 0.0004 & 0.0357 & 0.2713 \\
$v^{\prime}=4$ & 0.0000 & 0.0023 & 0.0418 \\
\hline \hline
\end{tabular}

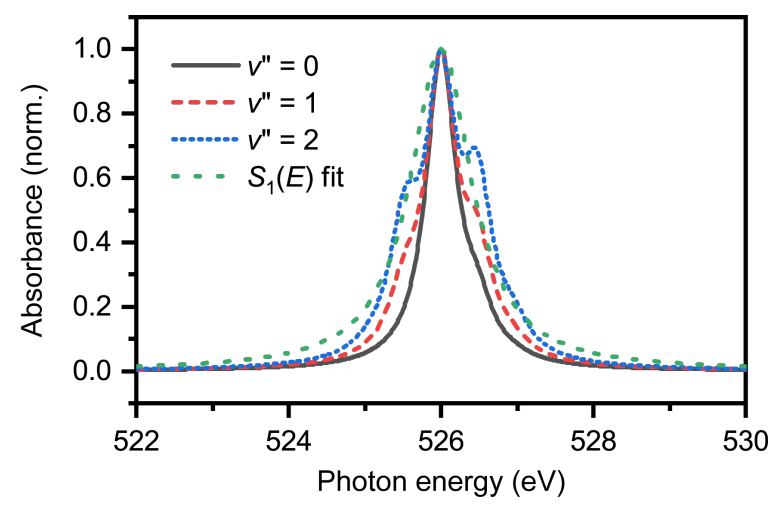

Figure S6: Calculated lineshapes for the $\mathrm{OH}$ radical in various vibrational states. The lineshape $S_{1}(E)$ that is obtained from the fit is also shown for comparison.

\section{Pre-edge shift}

In addition to observing the proton transfer and vibrational cooling dynamics of the $\mathrm{H}_{2} \mathrm{O}^{+}$ and $\mathrm{OH}$ radicals at $526 \mathrm{eV}$, we also observe a red-shift of the pre-edge on the sub-picosecond timescale (Fig. 2A). A single time constant $\tau$ effectively fits all the data over the entire probe photon energy range of 531.0 - $533.7 \mathrm{eV}$ (Fig. S7), as described in the following expression

$$
A(E, t)=S_{0}(E)+\left[S_{1}(E)+S_{2}(E) e^{-t / \tau}\right] H(t) * I R F(t) .
$$

The origin of this absorption increase warrants further investigation with a thinner liquid microjet target that permits measurements beyond the main absorption edge at $537 \mathrm{eV}$. We 


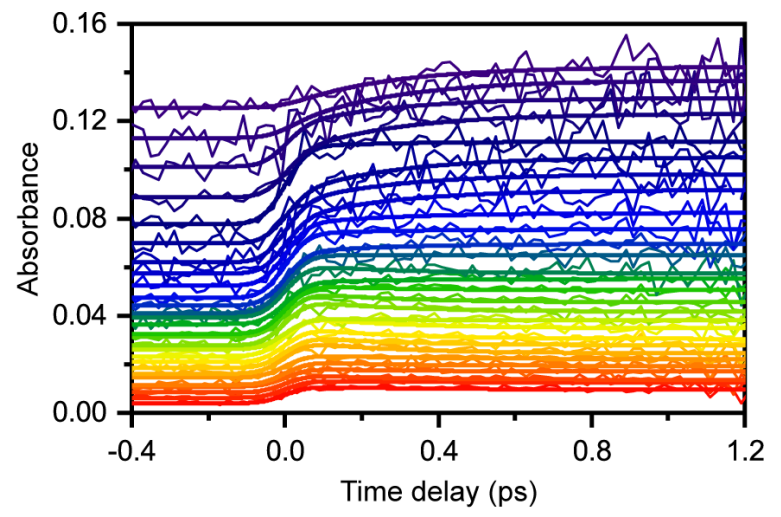

Figure S7: Differential absorption traces near the pre-edge resonance. The various traces, from red to violet, span $531.03 \mathrm{eV}$ (red) to $533.73 \mathrm{eV}$ (violet). All traces can be fit to a single time constant of $0.26 \pm 0.03 \mathrm{ps}$.

exclude the possibility that the observed dynamics at the pre-edge is due to the $\mathrm{H}_{2} \mathrm{O}^{+}$radical cation or the hot $\mathrm{OH}$ radical because the retrieved time constant $(0.26 \pm 0.03 \mathrm{ps})$ is significantly different from the sub-picosecond time constants observed at $526 \mathrm{eV}(46 \pm 10 \mathrm{fs}$ and $0.16 \pm 0.03$ ps) and our ab initio calculations (see Fig. 1B in the main text and Section 6 below) do not predict any $\mathrm{x}$-ray absorption signature due to $\mathrm{H}_{2} \mathrm{O}^{+} / \mathrm{OH}$ in the pre-edge region. We also exclude the $\mathrm{OH}^{-}$anion, which exhibits x-ray absorption resonances near the pre-edge (46), but the timescale for its formation due to geminate recombination is substantially longer, $\gtrsim 10$ ps. Hence, we posit that the spectral change observed at the pre-edge reflects the formation of the hydrated electron because of the agreement between the retrieved time constant and the 0.25-ps timescale for hydrated electron formation previously established by optical pumpprobe spectroscopy $(7,8)$. Hydrated electron formation is expected to disrupt the hydrogen bond network, which according to previous first-principles calculations (47) and infrared pump-soft $\mathrm{x}$-ray probe (48) measurements of liquid water, results in an increase in the pre-edge x-ray absorption. 


\section{Theoretical Methods}

Ab initio molecular dynamics To obtain an ab initio description of excited-state molecular dynamics of strong-field ionized water, we adopt a two-layered QM/MM approach, where a core region is treated quantum-mechanically $(\mathrm{QM})$, and is embedded in a larger region described classically (MM) (49). We describe the electronic structure of a $\left(\mathrm{H}_{2} \mathrm{O}\right)_{12}^{+}$cluster quantummechanically at the Hartree-Fock level of theory with the 6-31G Gaussian basis set. The QM cluster is embedded in 1012 additional water molecules described by the classical SPC/E water model (50). Internal bonds and angles in the MM region are constrained using the RATTLE algorithm (51). Within the QM/MM approach, the energy is given as

$$
E\left(\vec{X}_{\text {tot }}\right)=E_{\mathrm{QM}}\left(\vec{X}_{\mathrm{QM}}\right)+E_{\mathrm{MM}}\left(\vec{X}_{\text {tot }}\right)-E_{\mathrm{MM}}\left(\vec{X}_{\mathrm{QM}}\right),
$$

where $\vec{X}_{\text {tot }}$ comprises all nuclear coordinates and $\vec{X}_{\mathrm{QM}}$ the subset of nuclear coordinates in the QM region. The excited-state energies and gradients are obtained via Koopmans' theorem. For details, see Ref. (27).

To describe nuclear motion, we adopt Tully's fewest switches surface hopping (FSSH), which includes non-Born Oppenheimer effects (25). We sample over trajectories from a total of 107 snapshots that were obtained from long, thermostated trajectories. For each snapshot, we consider electronic states within the top $1.5 \mathrm{eV}$ of the valence $\mathrm{MO}$ band to account for strong-field ionization.

X-ray absorption spectra X-ray absorption spectra are calculated at the same level of electronic structure theory as used for the molecular dynamics. The absorption cross section is given as

$$
\sigma(\omega)=\frac{4}{3} \pi^{2} \omega \alpha \sum_{d=x, y, z}\left|\left\langle\phi_{f}|d| \phi_{i}\right\rangle\right|^{2} \mathcal{V}\left(\omega-\omega_{\text {res }}\right)
$$


where $\omega_{\text {res }}=\epsilon_{f}-\epsilon_{i}$. Here, $\epsilon_{i}$ and $\epsilon_{f}$ refer to the initial and final MO energies, $\left\langle\phi_{f}|d| \phi_{i}\right\rangle$ are the transition dipole matrix elements, and $\alpha$ is the fine structure constant. We take the natural line width and the $I R F$ into account with a Voigt profile $\mathcal{V}$, comprising a Lorentz function with a FWHM of $170 \mathrm{meV}$ and a Gaussian function with a FWHM of $100 \mathrm{meV}$. The 2D spectrum in Fig. 3A of the main text is additionally convolved in time with a Gaussian function of $10 \mathrm{fs}$ FWHM.

Identification of $\mathrm{OH}$ formation In order to identify the formation of $\mathrm{OH}$ in the trajectories, we employ bond-order analysis (28). The bond order of a given oxygen atom to its nearest two hydrogen atoms in the QM region is denoted as $b_{\mathrm{H} 1}, b_{\mathrm{H} 2}$, respectively. $\mathrm{In}_{2} \mathrm{O}$ and $\mathrm{H}_{2} \mathrm{O}^{+}$, we expect $b_{\mathrm{H} 1} \approx b_{\mathrm{H} 2}$. To derive a criterion for $\mathrm{OH}$ formation, we demand that $(i)$ the initial state is reproduced (no $\mathrm{OH}$ is present), (ii) the final proton transfer status after $200 \mathrm{fs}$ is reproduced, and (iii) at any given time step, there is at maximum one $\mathrm{OH}$ per trajectory. With this, we establish $\mathrm{OH}$ formation if

$$
b_{\mathrm{H} 2} / b_{\mathrm{H} 1}<0.45 \text {. }
$$

Identification of the $\mathrm{OH}$ allows us to study its diffusion towards the MM region (Fig. S8). While the radius of gyration,

$$
r_{\text {gyr }}^{2}=\frac{\sum_{k=1}^{N_{\text {atoms }}} m_{k}\left|\vec{r}_{k}-\vec{R}_{\mathrm{COM}}\right|^{2}}{\sum_{k=1}^{N_{\text {atoms }}} m_{k}},
$$

of the $\left(\mathrm{H}_{2} \mathrm{O}\right)_{12}^{+}$QM cluster varies little over the course of $200 \mathrm{fs}$, the distance of the $\mathrm{OH}$ to the center of mass $(\mathrm{COM})$ of the remaining $\mathrm{QM}$ region increases upon $\mathrm{OH}$ formation. Note that $\mathrm{OH}$ is formed initially close to the surface of the QM cluster. The $\mathrm{OH}$ to $\mathrm{COM}$ distance surpasses $r_{\text {gyr }}$ after $80 \mathrm{fs}$. This is accompanied by a linear drift in the spectrum peak position. Therefore, we conclude that this linear drift is an artefact of the limited QM cluster size that enforces $\mathrm{OH}$ diffusion. 


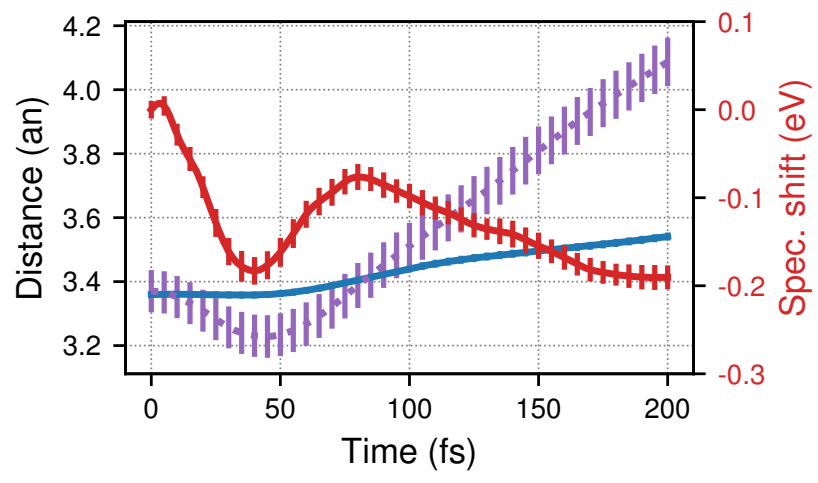

Figure S8: Analysis of distances within the $\left(\mathrm{H}_{2} \mathrm{O}\right)_{12}^{+}$cluster. Radius of gyration of the QM region (blue) and distance of the OH to the COM of the QM region (purple). The shift of the spectrum peak position is included (red).

Hole and charge center The distance between the hole center and the charge center is a means of quantifying the progress of the proton transfer (cf. Ref. (15)). The hole can by Koopmans' theorem be identified with a Hartree-Fock molecular orbital $\alpha$. Its center is given by

$$
\vec{R}_{H}^{(\alpha)}=\sum_{k=1}^{N_{\text {atoms }}} G_{k}^{(\alpha)} \vec{r}_{k},
$$

where $G_{k}^{(\alpha)}$ is the normalized weight of MO $\alpha$ at atom $k$. The charge center is located at

$$
\vec{R}_{C}=\sum_{k=1}^{N_{\text {atoms }}}\left(q_{k}^{(\mathrm{MC})}-q_{k}^{(\mathrm{WM})}\right) \vec{r}_{k},
$$

with the Mulliken charges $q_{k}^{(\mathrm{MC})}$ and the water model charges $q_{k}^{(\mathrm{WM})}$, where $q_{\mathrm{O}}^{(\mathrm{WM})}=-0.8476$, $q_{\mathrm{H}}^{(\mathrm{WM})}=+0.4238$. Since the charges on the $\mathrm{OH}$ are not adequately described within the water model, we exclude the $\mathrm{OH}$ from the summation in Eq. (S15).

Hole localization Hole localization for a valence hole in orbital $\alpha$ is measured by the hole radius $r_{H}^{(\alpha)}$, which is calculated analogously to a radius of gyration as

$$
r_{H}^{(\alpha)}=\sqrt{\sum_{k=1}^{N_{\text {atoms }}} G_{k}^{(\alpha)}\left|\vec{r}_{k}-\vec{R}_{H}^{(\alpha)}\right|^{2}} .
$$


Core and valence MOs The position of the absorption peak is governed by the energy difference between the participating molecular orbitals. Fig. S9 shows the density of valence and core molecular orbitals over time. The orbitals that dominate the absorption (located at $\mathrm{H}_{2} \mathrm{O}^{+} / \mathrm{OH}$ ) are highlighted by white lines. While the overall distribution of both valence and core orbitals broadens with time, there is a clear separation of the $\mathrm{OH}$ orbitals towards higher orbital energies $\varepsilon_{\mathrm{MO}}$. Since this shift is comparable for valence and core orbitals, the overall spectral shift is comparatively small with a maximum of about $20 \mathrm{meV}$.

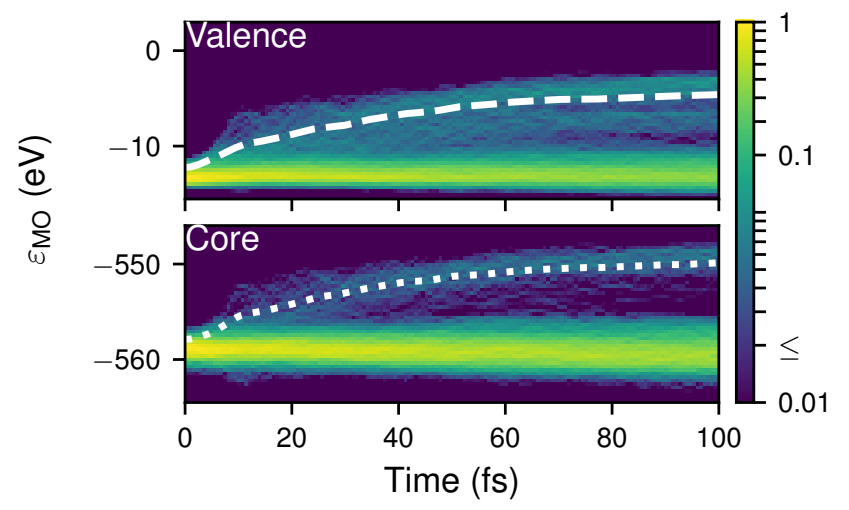

Figure S9: Density of valence (top) and core molecular orbitals (MOs) (bottom). MOs with dominant contribution to absorption spectrum highlighted (white lines).

Basis set effects The ab initio molecular dynamics simulations and $\mathrm{x}$-ray absorption spectra presented in the main text were obtained using the 6-31G Gaussian basis set. By adding diffuse functions to the basis set, as was done in the gas phase spectra of Fig. 1B of the main text, the description of $\mathrm{OH}$ orbitals is improved and thus the $\mathrm{x}$-ray absorption spectrum is affected. To study the effects of the basis size, while keeping the existing molecular dynamics trajectories, we calculated $\mathrm{x}$-ray absorption including diffuse functions within the $6-31+\mathrm{G}$ basis set. The resulting 2D spectrum is shown in Fig. S10. It corresponds to Fig. 3A of the main text and exhibits a blueshift of the spectrum at long times. 


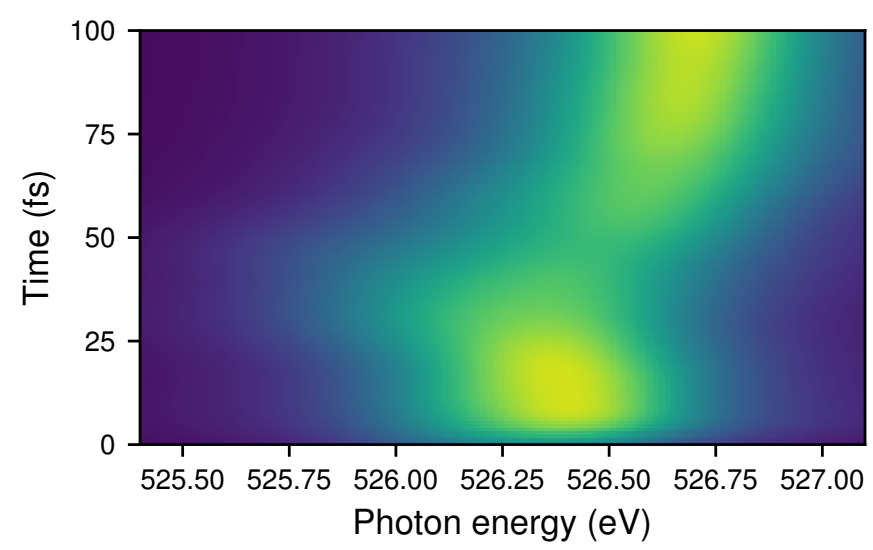

Figure S10: Two-dimensional x-ray absorption spectrum as in Fig. 3A of the main text, but calculated with the $6-31+\mathrm{G}$ basis set which includes diffuse basis functions.

In order to resolve the difference between Figs. 3A (main text) and S10, in Fig. S11, we study the effect of including diffuse functions for the x-ray absorption spectrum of $\mathrm{OH}$ and $\mathrm{H}_{2} \mathrm{O}^{+}$in the gas phase. We find that, while the $\mathrm{OH}$ absorption line is redshifted with respect to the $\mathrm{H}_{2} \mathrm{O}^{+}$absorption line with the $6-31 \mathrm{G}$ basis set, a blueshift is observed for the $6-31+\mathrm{G}$ basis set. This is consistent with the blueshift seen in Fig. S10 for long times, when proton transfer is completed and absorption is taking place mainly at the $\mathrm{OH}$.

With the computational limitations that come with the ab initio treatment of an $\left(\mathrm{H}_{2} \mathrm{O}\right)_{12}^{+} \mathrm{QM}$ cluster, molecular dynamics trajectories with the $6-31+\mathrm{G}$ basis set are not feasible. (Please note that, unlike e.g. Ref. (15), we include non-adiabatic effects in our simulations.) However, based on the comparison in Fig. S11, we find that the $\mathrm{x}$-ray absorption spectrum is sensitive to the decreasing distance between neighboring oxygen atoms (Fig. 3D, main text). The energy difference of about $1.0 \mathrm{eV}$ between the $\mathrm{x}$-ray absorption lines of $\mathrm{OH}$ in the $6-31 \mathrm{G}$ and the $6-31+\mathrm{G}$ basis, respectively, arises mainly from the lowering of the oxygen core orbital when diffuse functions are introduced. The valence orbital, which is relevant for the molecular dynamics, is less affected. 

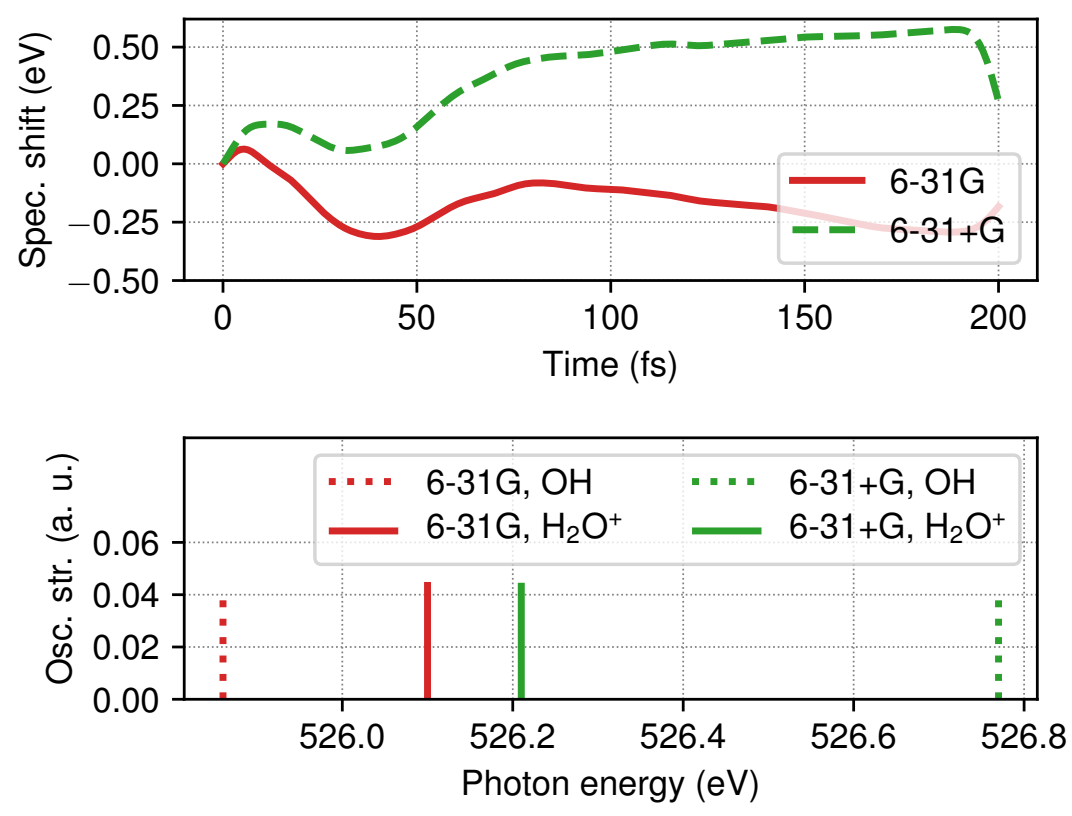

Figure S11: Comparison of $\mathrm{x}$-ray absorption spectra obtained using the 6-31G and 6-31+G basis sets. Top panel: Position of the x-ray absorption peak over time. Bottom panel: Gas phase spectra of $\mathrm{OH}$ and $\mathrm{H}_{2} \mathrm{O}^{+}$. 